\title{
Picard-Vessiot Extensions of Real Differential Fields
}

\author{
Teresa CRESPO ${ }^{\dagger}$ and Zbigniew HAJTO ${ }^{\ddagger}$ \\ ${ }^{\dagger}$ Departament de Matemàtiques i Informàtica, Universitat de Barcelona, \\ Gran Via de les Corts Catalanes 585, 08007 Barcelona, Spain \\ E-mail: teresa.crespo@ub.edu \\ $\ddagger$ Faculty of Mathematics and Computer Science, Jagiellonian University, \\ ul. Prof. S. Eojasiewicza 6, 30-348 Kraków, Poland \\ E-mail: zbigniew.hajto@uj.edu.pl
}

Received July 04, 2019, in final form December 22, 2019; Published online December 24, 2019

https://doi.org/10.3842/SIGMA.2019.100

\begin{abstract}
For a linear differential equation defined over a formally real differential field $K$ with real closed field of constants $k$, Crespo, Hajto and van der Put proved that there exists a unique formally real Picard-Vessiot extension up to $K$-differential automorphism. However such an equation may have Picard-Vessiot extensions which are not formally real fields. The differential Galois group of a Picard-Vessiot extension for this equation has the structure of a linear algebraic group defined over $k$ and is a $k$-form of the differential Galois group $H$ of the equation over the differential field $K(\sqrt{-1})$. These facts lead us to consider two issues: determining the number of $K$-differential isomorphism classes of Picard-Vessiot extensions and describing the variation of the differential Galois group in the set of $k$-forms of $H$. We address these two issues in the cases when $H$ is a special linear, a special orthogonal, or a symplectic linear algebraic group and conclude that there is no general behaviour.
\end{abstract}

Key words: real Picard-Vessiot theory; linear algebraic groups; group cohomology; real forms of algebraic groups

2010 Mathematics Subject Classification: 12H05; 13B05; 14P05; 12D15

\section{Introduction}

To a homogeneous linear differential equation defined over a differential field $K$ with field of constants $k$, Picard-Vessiot theory associates a differential field extension $L$ of $K$, differentially generated over $K$ by a fundamental system of solutions of the equation, and with constant field equal to $k$, called a Picard-Vessiot extension for the given equation. When $k$ is algebraically closed, Kolchin [18] established that a Picard-Vessiot extension for the given equation exists and is unique up to $K$-differential isomorphism. The differential Galois group DGal $(L / K)$ is defined as the group of $K$-differential automorphisms of $L$ and has the structure of a linear algebraic group defined over $k$.

For a homogeneous linear differential equation defined over a formally real differential field $K$ with real closed field of constants $k$, Crespo, Hajto and van der Put proved in [11], the existence and unicity up to $K$-differential isomorphism of a formally real Picard-Vessiot extension, endowed with an ordering extending the one in $K$. We note that such a linear differential equation may also have Picard-Vessiot extensions which are not formally real fields. Our result was later generalized in [15], by using model-theoretic methods, to the case when $K$ is a differential field of characteristic 0 such that its field of constants $k$ is existentially closed in $K$ for strongly normal extensions of $K$ associated to logarithmic differential equations over $K$ on algebraic groups over $k$. Let $L$ be a Picard-Vessiot extension of a formally real differential field $K$ with real closed field of constants $k$. Then the differential Galois group $G=\operatorname{DGal}(L \mid K)$ has the structure of a linear algebraic group defined over $k$ (see Section 2). 
Differential Galois theory over non-algebraically closed field of constants has been developed by several authors, see [1, 2, 19]. The inverse problem in this setting has also been considered (see $[4,13])$. In particular, Dyckerhoff proved in [13] that every linear algebraic group over $\mathbb{R}$ is a differential Galois group over the field $\mathbb{R}(x)$ of rational functions.

In Picard-Vessiot theory over formally real differential fields, one may find phenomena which do not arise in the context of differential fields with algebraically closed field of constants. Given a linear differential equation

$$
\mathcal{L}(Y):=Y^{(n)}+a_{n-1} Y^{(n-1)}+\cdots+a_{1} Y^{\prime}+a_{0} Y=0
$$

with $a_{n-1}, \ldots, a_{1}, a_{0}$ belonging to a formally real differential field $K$ with real closed field of constants $k$, one may ask the following questions which do not have a counterpart in the case when the field of constants $k$ is algebraically closed.

- How many $K$-differential isomorphism classes of Picard-Vessiot extensions are there for $\mathcal{L}(Y)=0$ ?

- Are the corresponding differential Galois groups $k$-isomorphic?

Concerning the first question, as mentioned above, it is known that there is at least one Picard-Vessiot extension of $K$ for $\mathcal{L}(Y)=0$, which is a formally real field. For the second one, let us note that if $L$ and $L^{\prime}$ are Picard-Vessiot extensions of a formally real differential field $K$ with real closed field of constants $k$ for the same equation, and $G=\operatorname{DGal}(L / K), G^{\prime}=\operatorname{DGal}\left(L^{\prime} / K\right)$ are the corresponding differential Galois groups, then we have $G \times_{k} \bar{k} \simeq G^{\prime} \times_{k} \bar{k}$, i.e., $G$ and $G^{\prime}$ are both $k$-forms of the group $H=G \times_{k} \bar{k}$.

In this paper, we consider a formally real Picard-Vessiot extension $L$ of $K$ for a linear differential equation $\mathcal{L}(Y)=0$ defined over $K$ and the differential Galois group $G=\operatorname{DGal}(L \mid K)$. We give an answer to the above questions in the case in which $H:=G \times_{k} \bar{k}$ is a special linear, special orthogonal, or symplectic linear algebraic group. Let us note that it makes sense to start with such a Picard-Vessiot extension $L / K$ since in [11, Proposition 3.3] Crespo, Hajto and van der Put proved that when the differential field $K$ is real closed, given a connected semisimple linear algebraic group $G$ defined over $k$, there exists a linear differential equation defined over $K$ and a formally real Picard-Vessiot extension $L \mid K$ for it such that $G=\operatorname{DGal}(L \mid K)$. The inspection of the different cases shows that there is no general pattern. The differential Galois group may be the same for all Picard-Vessiot extensions of $\mathcal{L}(Y)=0$ or range over the whole set of $k$-forms of $H$. For a linear differential equation defined over a formally real differential field $K$ the determination of its real differential group $G$ gives more information on the behaviour of the solutions than the determination of the complexification $H$ of $G$. For example, for $k=\mathbb{R}$ and $K$ a field of real functions, the determination of $G$ will give information on the existence of oscillating functions among the solutions of $\mathcal{L}(Y)=0$ (see [9] and the earlier topological approach in [14]). It is then interesting to study how the real differential group $\operatorname{DGal}(L \mid K)$ varies as $L$ runs over the $K$-isomorphism classes of Picard-Vessiot extensions.

We refer the reader to [8] for the topics on differential Galois theory, when the field of constants is algebraically closed, to [6] or [21] for those on formally real fields and to [20, 24, 25] for those on linear algebraic groups.

\section{Preliminaries}

For the reader's convenience, we recall the definitions of formally real field, real closed field and Picard-Vessiot extension.

Definition 2.1. A formally real field is a field which may be given an ordering compatible with the field operations. Equivalently, a field $K$ is formally real if -1 is not a sum of squares in $K$. 
A formally real field is real closed if it has no nontrivial algebraic extensions which are formally real fields. Equivalently, a field $k$ is real closed if -1 is not a square in $k$ and $k(\sqrt{-1})$ is algebraically closed.

We note that in the literature on real algebraic geometry "real field" is frequently used for "formally real field". A field of positive characteristic is not formally real. The field $\mathbb{R}$ of real numbers is the standard example of a real closed field. The field $\mathbb{R}(x)$ of rational functions and the field of formal Laurent series $\mathbb{R}(x)$ with derivation $\mathrm{d} / \mathrm{d} x$ are examples of real differential fields with real closed field of constants.

Definition 2.2. Given a linear differential equation

$$
\mathcal{L}(Y):=Y^{(n)}+a_{n-1} Y^{(n-1)}+\cdots+a_{1} Y^{\prime}+a_{0} Y=0
$$

defined over a differential field $K$, with field of constants $k$, a Picard-Vessiot extension of $K$ for $\mathcal{L}(Y)=0$ is a differential field extension $L \mid K$ such that

a) $L$ is differentially generated over $K$ by a full set of solutions of $\mathcal{L}(Y)=0$;

b) the field of constants of $L$ is $k$.

Let us assume that $K$ is a formally real differential field with real closed field of constants $k$, $\mathcal{L}(Y)=0$ a linear differential equation defined over $K$ and $L \mid K$ a Picard-Vessiot extension for $\mathcal{L}(Y)=0$. In this case, we note that the set $\operatorname{DHom}_{K}(L, L(\mathrm{i}))$ of $K$-differential morphisms from $L$ to $L(\mathrm{i})$ is in bijection with the set $\mathrm{DAut}_{K(\mathrm{i})} L(\mathrm{i})$ of $K(\mathrm{i})$-differential automorphisms of $L(\mathrm{i})$. We define the differential Galois group of $L \mid K$ as the set $\operatorname{DHom}_{K}(L, L(\mathrm{i}))$ with the group structure obtained by transferring the one of $\mathrm{DAut}_{K(\mathrm{i})} L(\mathrm{i})$ via the above bijection. The differential Galois group has the structure of a $k$-defined linear algebraic group (see [10, Proposition 4.1]). We note that the proof of the existence of a formally real Picard-Vessiot extension for a linear differential equation defined over a formally real differential field with real closed field of constants given in [10, Theorems 3.2 and 3.3 and Corollary 3.4] is not right. The remaining proofs in [10] are correct.

In the sequel, $K$ will denote a formally real differential field with real closed field of constants $k, \mathcal{L}(Y)=0$ a linear differential equation defined over $K, L \mid K$ a formally real PicardVessiot extension for $\mathcal{L}(Y)=0$ and $G$ the differential Galois group of $L \mid K$. We want to determine the number of Picard-Vessiot extensions of $K$ for $\mathcal{L}(Y)=0$, up to $K$-differential isomorphism, and the differential Galois group of each of them.

The set of $K$-differential isomorphism classes of Picard-Vessiot extensions for $\mathcal{L}(Y)=0$ is in one-to-one correspondence with the cohomology set $H^{1}(k, G(\bar{k}))$, where $\bar{k}$ denotes the algebraic closure of $k$ and $G$ denotes the differential Galois group DGal $(L / K)$. Indeed, we have a bijection between the set of $K$-differential isomorphism classes of Picard-Vessiot extensions for $\mathcal{L}(Y)=0$ and the set of isomorphism classes of fiber functors $\omega:\langle M\rangle_{\otimes} \rightarrow \operatorname{vect}(k)$, where $\langle M\rangle_{\otimes}$ denotes the Tannakian category generated by the $K$-differential module $M$ associated to $\mathcal{L}(Y)=0$ [11, Proposition 1]. In turn, this set of isomorphism classes of fiber functors is in bijection with the set of isomorphism classes of $G$-torsors, by [12, Theorem 3.2]. Finally, the set of isomorphism classes of $G$-torsors is in bijection with $H^{1}(k, G(\bar{k}))$ (see, e.g., [27, Lemma A.5.1]). If $L^{\prime}$ is a Picard-Vessiot extension for $\mathcal{L}(Y)=0, L(\mathrm{i})$ and $L^{\prime}(\mathrm{i})$ are Picard-Vessiot extensions of $K(\mathrm{i})$ for $\mathcal{L}(Y)=0$. Since the field of constants of $K(\mathrm{i})$ is $\bar{k}$, we have an isomorphism of differential fields $f: L(\mathrm{i}) \rightarrow L^{\prime}(\mathrm{i})$, by the unicity of the Picard-Vessiot extension in the case when the field of constants is algebraically closed. The group Gal $(\bar{k} \mid k)$ acts on the set of isomorphisms from $L(\mathrm{i})$ to $L^{\prime}(\mathrm{i})$ by $s(f)=s \circ f \circ s^{-1}$, for $s \in \operatorname{Gal}(\bar{k} \mid k)$, where we denote also by $s$ the automorphisms of $L(\mathrm{i})$ and $L^{\prime}(\mathrm{i})$ induced by $s$. The 1-cocycle $x$ corresponding to $L^{\prime}$ is determined by $x(c)=f^{-1} \circ c(f)$, where $c$ is the nontrivial element in Gal $(\bar{k} \mid k)$. Reciprocally, 
if $x$ is a 1-cocycle from Gal $(\bar{k} \mid k)$ to $G(\bar{k})$, then the corresponding Picard-Vessiot extension corresponding to $x$ is the subfield of $L(\mathrm{i})$ fixed by the automorphism $x(c) \circ c$, by Galois descent theory (see [23, Chapter III, Section 1.3]). The Galois group Gal $(\bar{k} \mid k)$ acts on $G(\bar{k})$, by an involution leaving $G$ invariant, hence the cohomology set $H^{1}(k, G(\bar{k}))$ depends on the $k$-form $G$ of $H=G \times{ }_{k} \bar{k}$.

Let now $G$ denote a linear algebraic group defined over a real closed field $k$ and let $H=$ $G \times{ }_{k} \bar{k}$. The set of $k$-forms of $H$ is in one-to-one correspondence with the cohomology set $H^{1}(k$, Aut $G(\bar{k}))$, where Gal $(\bar{k} \mid k)$ acts on Aut $G(\bar{k})$ by $s(f)=s \circ f \circ s^{-1}$, for $s \in \operatorname{Gal}(\bar{k} \mid k)$, $f \in \operatorname{Aut} G(\bar{k})$, as usual. Let us note that the classification of the $k$-forms of $H$ is equivalent to the classification of the real forms of the corresponding complex group.

We consider the map

$$
\Phi: H^{1}(k, G(\bar{k})) \rightarrow H^{1}(k, \operatorname{Aut} G(\bar{k}))
$$

induced by the morphism from $G(\bar{k})$ to Aut $G(\bar{k})$ sending an element $g$ in $G(\bar{k})$ to conjugation by $g$. When $G$ is the differential Galois group of a Picard-Vessiot extension $L$ of $K$ for a linear differential equation $\mathcal{L}(Y)=0$, $\Phi$ sends the element in $H^{1}(k, G(\bar{k}))$ corresponding to a PicardVessiot extension $L^{\prime}$ of $K$ for $\mathcal{L}(Y)=0$ to the element in $H^{1}(k$, Aut $G(\bar{k}))$ corresponding to $\operatorname{DGal}\left(L^{\prime} \mid K\right)$ (see [11, Section 3, Observations 1]).

For $c$ the nontrivial element of $\operatorname{Gal}(\bar{k} \mid k)$, we write $\bar{a}=c(a)$, for $a$ an element in $\bar{k}$. For $v=\left(a_{1}, \ldots, a_{n}\right) \in \bar{k}^{n}$, we shall write $\bar{v}=\left(\overline{a_{1}}, \ldots, \overline{a_{n}}\right)$ and for $M=\left(a_{i j}\right)$ a matrix with entries in $\bar{k}, \bar{M}=\left(\overline{a_{i j}}\right)$.

We shall consider a linear algebraic group $G$ defined over the real closed field $k$, such that $H=$ $G \times_{k} \bar{k}$ is either a special linear group $\mathrm{SL}(n)$, a special orthogonal group $\mathrm{SO}(n)$ or a symplectic group $\operatorname{Sp}(n)$. We will then consider the real forms of each of these groups. We refer to [23] or [16] for their determination, to [7] or [26] for a more explicit description of them.

We will determine in each case the number of $K$-differential isomorphism classes of PicardVessiot extensions of $K$ for $\mathcal{L}(Y)=0$ and the differential Galois group for each class.

Let $\mathrm{i}$ denote a square root of -1 in $\bar{k}$. For $p, n$ integers with $0 \leq p \leq n$, we define the $n \times n$ matrices

$$
I_{p}=\left(\begin{array}{cc}
\operatorname{Id}_{p} & 0 \\
0 & -\operatorname{Id}_{n-p}
\end{array}\right), \quad J_{p}=\left(\begin{array}{cc}
\operatorname{Id}_{p} & 0 \\
0 & \mathrm{i} \operatorname{Id}_{n-p}
\end{array}\right)
$$

\section{Forms of SL $(n)$}

The real forms of $\mathrm{SL}(n), n \geq 2$, are

1) $\operatorname{SL}(n, k)$;

2) $\operatorname{SL}(n / 2, \mathbb{H})$ if $n$ is even, where $\mathbb{H}$ denotes the quaternion algebra over $k$;

3) $\mathrm{SU}(n, \bar{k}, h)$, where $h$ is a nondegenerate hermitian form on $\bar{k}^{n}$.

For $G$ each of these real forms and $K$ a formally real differential field, with real closed field of constants $k$, we consider a linear differential equation $\mathcal{L}(Y)=0$ of order $n$ defined over $K$ and a Picard-Vessiot extension $L \mid K$ for $\mathcal{L}(Y)=0$ such that $L$ is formally real and $\operatorname{DGal}(L / K) \simeq G$.

\section{1 $G=\mathrm{SL}(n, k)$}

We have $\left|H^{1}(k, G(\bar{k}))\right|=1$ [22, Chapter X, Section 1], hence $L \mid K$ is the unique Picard-Vessiot extension for $\mathcal{L}(Y)=0$, up to $K$-differential isomorphism. 


\section{$3.2 G=\mathrm{SL}(n / 2, \mathbb{H})$}

Let us denote by $1, I, J, K$ the basis elements of $\mathbb{H}$. We recall that $\operatorname{GL}(n / 2, \mathbb{H})$ embeds into GL $(n, \bar{k})$ via the morphism $\left(h_{i j}\right) \mapsto\left(\mu\left(h_{i j}\right)\right)$, where

$$
\mu(a+b I+c J+d K)=\left(\begin{array}{cc}
a+b \mathrm{i} & c+d \mathrm{i} \\
-c+d \mathrm{i} & a-b \mathrm{i}
\end{array}\right), \quad a, b, c, d \in k .
$$

We denote by $A_{n}$ the matrix $\left(a_{i j}\right)_{1 \leq i, j \leq n}$ with

$$
a_{i j}=\left\{\begin{aligned}
1 & \text { if } i \text { is odd and } j=i+1 \\
-1 & \text { if } i \text { is even and } j=i-1 \\
0 & \text { in all other cases. }
\end{aligned}\right.
$$

We have $\mu(\mathrm{GL}(n / 2, \mathbb{H}))=\left\{M \in \mathrm{GL}(n, \bar{k}): M=A_{n} \bar{M} A_{n}^{-1}\right\}$ and $\mu(\mathrm{SL}(n / 2, \mathbb{H}))=\mathrm{SL}(n, \bar{k}) \cap$ $\mu(\mathrm{GL}(n / 2, \mathbb{H}))$.

By [17, Chapter VII, Section 29, Corollary 29.4], we have $H^{1}(k, G(\bar{k})) \simeq k^{*} / \operatorname{Nrd}\left(\mathbb{H}^{n / 2}\right)$. Since the norm of a quaternion is always positive, we obtain $\left|H^{1}(k, G(\bar{k}))\right|=2$ (see also [23, Chapter III, Section 1.4]). We have then two Picard-Vessiot extensions for $\mathcal{L}(Y)=0$, up to $K$ differential isomorphism. A nontrivial 1-cocycle $x$ of Gal $(\bar{k} \mid k)$ in SL $(n, \bar{k})$ is given by $x(c)=\zeta \mathrm{Id}$, for $\zeta$ a primitive $n$-th root of unity. A $K$-differential automorphism of $L(\mathrm{i})$ corresponding to $x$ is given by the matrix $A:=\zeta^{-1 / 2}$ Id on the vector space of solutions, since $A$ satisfies $A^{-1} c(A)=x(c)$ (see [22, Chapter X, Section 2, Proposition 4]). Conjugation by $\zeta^{-1 / 2}$ Id leaves the group $\mathrm{SL}(n / 2, \mathbb{H})$ stable. We obtain that the Picard-Vessiot extensions for $\mathcal{L}(Y)=0$ in both $K$-differential isomorphy classes have $\mathrm{SL}(n / 2, \mathbb{H})$ as differential Galois group.

\section{$3.3 \quad G=\mathrm{SU}(\boldsymbol{n}, \bar{k}, \boldsymbol{h})$}

It is known that if $h$ is a nondegenerate hermitian form on $\bar{k}^{n}$, then $h$ is equivalent to a hermitian form with matrix $I_{p}$, for some integer $p$ with $0 \leq p \leq n$, called the index of $h$ and that two nondegenerate hermitian forms on $\bar{k}^{n}$ are equivalent if and only if their indices coincide (see [17, Chapter VII, Section 29, Example 29.19] and [5, Section 3.3]).

We fix $G=\left\{M \in \mathrm{SL}(n, \bar{k}): \bar{M}^{t} I_{p} M=I_{p}\right\}$ and consider the action of Gal $(\bar{k} \mid k)$ on SL $(n, \bar{k})$ given by $c(M)=I_{p}\left(\bar{M}^{t}\right)^{-1} I_{p}$. We shall prove

$$
\left|H^{1}(k, G(\bar{k}))\right|= \begin{cases}{\left[\frac{n}{2}\right]+1,} & \text { when } n \text { is odd or } p \text { is even, } \\ \frac{n}{2}, & \text { when } n \text { is even and } p \text { is odd. }\end{cases}
$$

To this end we shall determine a maximal set of pairwise nonequivalent 1-cocycles from $\operatorname{Gal}(\bar{k} \mid k)$ in SL $(n, \bar{k})$. For such a cocycle $x$, we may assume that the image of $\mathrm{Id} \in \mathrm{Gal}(\bar{k} \mid k)$ is the identity matrix and then $x$ is determined by the image $B \in \mathrm{SL}(n, \bar{k})$ of the unique non trivial element $c$ in Gal $(\bar{k} \mid k)$. By the 1-cocycle condition, $B$ must satisfy $B c(B)=\mathrm{Id}$. We denote by $x_{q}$ the cocycle given by $c \mapsto B_{q}$, where $B_{q}=I_{q} I_{p}$, with $q$ an integer of the same parity as $p$ and $0 \leq q \leq n$. Let us see that every 1-cocycle $x$ from $\operatorname{Gal}(\bar{k} \mid k)$ in $\mathrm{SL}(n, \bar{k})$ is equivalent to some $x_{q}$. As said above, such a cocycle $x$ is determined by $x(c)=B$ satisfying $B c(B)=\mathrm{Id}$, i.e., $B I_{p}\left(\bar{B}^{t}\right)^{-1} I_{p}=\mathrm{Id}$, equivalently $B I_{p}=I_{p} \bar{B}^{t}=\left(\overline{B I_{p}}\right)^{t}$, so $B I_{p}$ is an hermitian

matrix, hence there exists an invertible matrix $M$ such that $M^{-1} B I_{p}\left(\bar{M}^{t}\right)^{-1}=I_{r}$, for some integer $r, 0 \leq r \leq n$. Equivalently

$$
M^{-1} B c(M)=I_{r} I_{p}
$$


Let us note that, taking determinants in (3.3), we obtain $\operatorname{det} M \overline{\operatorname{det} M}=1$, hence there exists $\zeta \in \bar{k}$ such that $\operatorname{det}(\zeta M)=1$ and $\zeta M$ satisfies (3.3). We have then that there exists a matrix $M \in \mathrm{SL}(n, \bar{k})$ satisfying (3.3), which means that $x$ is equivalent to the 1-cocycle $x_{r}$ determined by $c \mapsto I_{r} I_{p}$. Since $B \in \mathrm{SL}(n, \bar{k})$, we have $\operatorname{det}\left(I_{r} I_{p}\right)=1$, so $r$ is an integer of the same parity as $p$.

Let us see now that the 1-cocycles $x_{q}$ are pairwise nonequivalent. We have $x_{q} \sim x_{q^{\prime}} \Leftrightarrow \exists M \in$ SL $(n, \bar{k})$ such that $B_{q^{\prime}}=M^{-1} B_{q} c(M)$. This equality is equivalent to $M I_{q^{\prime}} \bar{M}^{t}=I_{q}$ which implies $q=q^{\prime}$, so the 1-cocycles $x_{q}$ are pairwise nonequivalent. We have then $H^{1}(k, G(\bar{k}))=$ $\left\{\left[x_{q}\right]: 0 \leq q \leq n, q \equiv p(\bmod 2)\right\}$. Then $\left|H^{1}(k, G(\bar{k}))\right|=|\{q \in \mathbb{Z}: 0 \leq q \leq n, q \equiv p(\bmod 2)\}|$ and we obtain the values in (3.2).

We have $Z(\mathrm{SL}(n, \bar{k}))=\mu_{n}(\bar{k})$. We want to determine the image of $\left[x_{q}\right]$ under the map $\Phi: H^{1}(k, G(\bar{k})) \rightarrow H^{1}(k$, Aut $G(\bar{k}))$. The 1-cocycle $x_{q}$ corresponds to a Picard-Vessiot extension $L_{q}$ of $K$ for $\mathcal{L}(Y)=0$ such that there is a differential isomorphism $f_{q}$ from $L(\mathrm{i})$ to $L_{q}(\mathrm{i})$ satisfying $x_{q}=f_{q}^{-1} c\left(f_{q}\right)$. The differential isomorphism $f_{q}$ is determined by the matrix $D_{q}$ giving the images of a vector space of solutions. The isomorphism $f_{q}$ satisfies $x_{q}=f_{q}^{-1} c\left(f_{q}\right)$ if and only if the matrix $D_{q}$ satisfies $B_{q}=D_{q}^{-1} \overline{D_{q}}$. We may take $D_{q}:=J_{q} J_{p}$. Since conjugation by $D_{q}$ leaves the group $G$ invariant, we obtain that all Picard-Vessiot extensions of $K$ for $\mathcal{L}(Y)=0$ have the same differential Galois group $G$.

Gathering the results in this section we may state the following theorem.

Theorem 3.1. Let $K$ be a formally real differential field with real closed field of constants $k$, $\mathcal{L}(Y)=0$ a linear differential equation defined over $K, L \mid K$ a formally real Picard-Vessiot extension for $\mathcal{L}(Y)=0$ and $G$ the differential Galois group of $L \mid K$. We assume that $G$ is a real form of $\mathrm{SL}(n)$.

(1) If $G=\mathrm{SL}(n, k), L \mid K$ is the unique Picard-Vessiot extension for the equation $\mathcal{L}(Y)=0$, up to $K$-differential isomorphism.

(2) If $G=\mathrm{SL}(n / 2, \mathbb{H})$, there are two Picard-Vessiot extensions for the equation $\mathcal{L}(Y)=0$, up to $K$-differential isomorphism, and both of them have differential Galois group $G$.

(3) If $G=\mathrm{SU}(n, \bar{k}, h)$, there are $[n / 2]+1$ (resp. [n/2]) Picard-Vessiot extensions for the equation $\mathcal{L}(Y)=0$, if $n$ is odd or $p$ is even (resp. if $n$ is even and $p$ is odd), up to $K$-differential isomorphism, and all of them have differential Galois group $G$.

\section{Forms of $\mathrm{SO}(n)$}

The real forms of $\mathrm{SO}(n)$, with $n$ odd, are the groups $\mathrm{SO}(n, k, Q)$, where $Q$ is a nondegenerate quadratic form on $k^{n}$. When $G$ is one of these forms, we proved in [11, Section 3, Examples 1 and 3] that the map $\Phi: H^{1}(k, G(\bar{k})) \rightarrow H^{1}(k$, Aut $G(\bar{k}))$ is a bijection. We consider now the case when $n$ is even.

The real forms of $\mathrm{SO}(n)$, with $n$ even, are

1) $\mathrm{SO}(n, k, Q)$, where $Q$ is a nondegenerate quadratic form on $k^{n}$;

2) $\mathrm{SU}(n / 2, \mathbb{H}, h)$, where $h$ is a nondegenerate anti-hermitian form on $\mathbb{H}^{n / 2}$ (with respect to the involution $\sigma$ of $\mathbb{H}$ defined by $a+b I+c J+d K \mapsto a-b I-c J-d K)$.

For $G$ each of these real forms and $K$ a formally real differential field, with real closed field of constants $k$, we consider a linear differential equation $\mathcal{L}(Y)=0$ of order $n$ defined over $K$ and a Picard-Vessiot extension $L \mid K$ for $\mathcal{L}(Y)=0$ such that $L$ is formally real and $\operatorname{DGal}(L / K) \simeq G$. 


\section{$4.1 \quad G=\operatorname{SO}(n, k, Q)$}

The quadratic form $Q$ is equivalent to a quadratic form with matrix $I_{p}$, for some integer $p$ with $0 \leq p \leq n$, which determines the equivalence class of $Q$.

The cohomology set $H^{1}(k, G(\bar{k}))$ is in one-to-one correspondence with the set of equivalence classes of quadratic forms on $k^{n}$ of rank $n$ and index of the same parity as $p$ (see [17, Chapter VII, Section 29, formula (29.29)]). We have then

$$
\left|H^{1}(k, G(\bar{k}))\right|= \begin{cases}\frac{n}{2}+1, & \text { when } p \text { is even, } \\ \frac{n}{2}, & \text { when } p \text { is odd. }\end{cases}
$$

The cocycles $x_{q}$ defined by $c \mapsto B_{q}$, where $B_{q}=I_{q} I_{p}$, with $q$ an integer of the same parity as $p$ and $0 \leq q \leq n$, form a complete system of representatives of the cohomology set $H^{1}(k, G(\bar{k}))$. The 1-cocycle $x_{q}$ corresponds to a Picard-Vessiot extension $L_{q}$ of $K$ for $\mathcal{L}(Y)=0$ such that there is a differential isomorphism $f_{q}$ from $L(\mathrm{i})$ to $L_{q}(\mathrm{i})$ satisfying $x_{q}=f_{q}^{-1} c\left(f_{q}\right)$. The differential isomorphism $f_{q}$ is determined by the matrix $D_{q}$ giving the images of a vector space of solutions. The isomorphism $f_{q}$ satisfies $x_{q}=f_{q}^{-1} c\left(f_{q}\right)$ if and only if the matrix $D_{q}$ satisfies $B_{q}=D_{q}^{-1} \overline{D_{q}}$. We may take $D_{q}:=J_{q} J_{p}$. If the matrix $M$ satisfies $M^{t} I_{p} M=I_{p}$, the conjugate matrix $N:=$ $D_{q} M D_{q}^{-1}$ satisfies $N^{t} I_{q} N=I_{q}$, hence the Picard-Vessiot extension corresponding to the 1cocycle $x_{q}$ has differential Galois group $\mathrm{SO}\left(n, k, Q_{q}\right)$, where $Q_{q}$ denotes the quadratic form with index $q$. Let us note that $\mathrm{SO}\left(n, k, Q_{q}\right)=\mathrm{SO}\left(n, k, Q_{n-q}\right)$, hence the Picard-Vessiot extension corresponding to $x_{q}$ and $x_{n-q}, 0 \leq q \leq(n / 2)-1$, have the same differential Galois group.

\section{$4.2 G=\mathrm{SU}(n / 2, \mathbb{H}, h), h$ anti-hermitian}

We have $\mathrm{U}(n / 2, \mathbb{H}, h)=\left\{M \in \mathrm{GL}(n / 2, \mathbb{H}): \sigma(M)^{t}[h] M=[h]\right\}$, for $[h]$ the matrix of the antihermitian form $h$, in some basis of $\mathbb{H}^{n / 2}$. The group $\mathrm{U}(n / 2, \mathbb{H}, h)$ is the group of automorphisms of the anti-hermitian vector space $\left(\mathbb{H}^{n / 2}, h\right)$. The set of equivalence classes of nondegenerate anti-hermitian forms over $\mathbb{H}^{n / 2}$ is in one-to-one correspondence with the cohomology set $H^{1}(k, \mathrm{U}(n / 2, \mathbb{H}, h)(\bar{k}))$. Up to equivalence, there is one single anti-hermitian form on $\mathbb{H}^{n}$, hence $H^{1}(k, \mathrm{U}(n / 2, \mathbb{H}, h)(\bar{k}))=1$.

We consider the exact sequence

$$
1 \rightarrow \mathrm{SU} \rightarrow \mathrm{U} \rightarrow \mu_{2} \rightarrow 1
$$

Since the reduced norm of a quaternion is always positive, the reduced norm $\mathrm{U}(n / 2, \mathbb{H}, h)(k) \rightarrow$ $\mu_{2}(k)$ is the trivial map. We obtain then for the cohomology sets the exact sequence

$$
1 \rightarrow \mu_{2} \rightarrow H^{1}(k, \mathrm{SU}(n / 2, \mathbb{H}, h)(\bar{k})) \rightarrow H^{1}(k, \mathrm{U}(n / 2, \mathbb{H}, h)(\bar{k}))
$$

Therefore $\left|H^{1}(k, \mathrm{SU}(n / 2, \mathbb{H}, h)(\bar{k}))\right|=2$. We obtain then that there are two Picard-Vessiot extensions for $\mathcal{L}(Y)=0$, up to $K$-differential isomorphism. We denote by $L^{\prime}$ the non formally real one. We may check that $\mu(G)$ is the intersection of (a conjugate form of) SO $(n, \bar{k})$ with $\mu(\mathrm{GL}(n / 2, \mathbb{H}))$. A nontrivial 1-cocycle of $\mathrm{Gal}(\bar{k} \mid k)$ in $G(\bar{k})$ is given by $c \mapsto A_{n}$, for $A_{n}$ the matrix defined by (3.1). The matrix $B=\left(b_{i j}\right)$ defined by

$$
b_{i j}=\left\{\begin{array}{cl}
\frac{1}{\sqrt{2}} & \text { if } i=j \text { or } i \text { is even and } j=i-1, \\
-\frac{1}{\sqrt{2}} & \text { if } i \text { is odd and } j=i+1 \\
0 & \text { in all other cases. }
\end{array}\right.
$$


satisfies $B^{-1} c(B)=A_{n}$, hence the cohomology class $[x]$ corresponds to the isomorphism class of the differential isomorphism $f$ from $L(\mathrm{i})$ to $L^{\prime}(\mathrm{i})$ with matrix $B$ on the vector space of solutions. Since conjugation by $B$ leaves $G$ invariant, we obtain that both Picard-Vessiot extensions have the same differential Galois group.

Gathering the results in this section we may state the following theorem. For completeness, we include the case $n$ odd.

Theorem 4.1. Let $K$ be a formally real differential field with real closed field of constants $k$, $\mathcal{L}(Y)=0$ a linear differential equation defined over $K, L \mid K$ a formally real Picard-Vessiot extension for $\mathcal{L}(Y)=0$ and $G$ the differential Galois group of $L \mid K$. We assume that $G$ is a real form of $\mathrm{SO}(n)$.

If $n$ is odd, there are $(n+1) / 2$ Picard-Vessiot extensions for the equation $\mathcal{L}(Y)=0$, up to $K$-differential isomorphism, and their differential Galois groups range over the whole set of real forms of $\mathrm{SO}(n)$.

Assume $n$ even.

(1) If $G=\mathrm{SO}\left(n, k, Q_{p}\right)$, where $Q_{p}$ is a nondegenerate quadratic form on $k^{n}$, of index $p$, there are $(n / 2)+1$ (resp. $n / 2)$ Picard-Vessiot extensions for the equation $\mathcal{L}(Y)=0$, up to $K$ differential isomorphism, when $p$ is even (resp. when $p$ is odd) and their differential Galois groups range over the whole set of groups $G=\mathrm{SO}\left(n, k, Q_{q}\right)$, with $Q_{q}$ a nondegenerate quadratic form on $k^{n}$, of index $q, 0 \leq q \leq n / 2$ and $q$ of the same parity as $p$.

(2) If $G=\mathrm{SU}(n / 2, \mathbb{H}, h)$, where $h$ is a nondegenerate anti-hermitian form on $\mathbb{H}^{n}$, there are two Picard-Vessiot extensions for the equation $\mathcal{L}(Y)=0$, up to $K$-differential isomorphism, and they have both differential Galois group $G$.

\section{Forms of $\operatorname{Sp}(2 n)$}

The real forms of $\operatorname{Sp}(2 n)$ are

1) $\operatorname{Sp}(2 n, k)$;

2) $\mathrm{SU}(n, \mathbb{H}, h)$, where $h$ is a nondegenerate hermitian form on $\mathbb{H}^{n}$ (with respect to the involution $\sigma$ of $\mathbb{H}$ defined by $a+b I+c J+d K \mapsto a-b I-c J-d K)$.

For $G$ each of these real forms and $K$ a formally real differential field, with real closed field of constants $k$, we consider a linear differential equation $\mathcal{L}(Y)=0$ of order $n$ defined over $K$ and a Picard-Vessiot extension $L \mid K$ for $\mathcal{L}(Y)=0$ such that $L$ is formally real and $\operatorname{DGal}(L / K) \simeq G$.

\section{$5.1 G=\operatorname{Sp}(2 n, k)$}

We have $\left|H^{1}(k, G(\bar{k}))\right|=1$ [22, Chapter X, Section 2, Corollary 2], hence $L \mid K$ is the unique Picard-Vessiot extension for $\mathcal{L}(Y)=0$, up to $K$-differential isomorphism.

\section{2 $G=\mathrm{SU}(n, \mathbb{H}, h), h$ hermitian}

If $h$ is a nondegenerate hermitian form on $\mathbb{H}^{n}$, then $h$ is equivalent to a hermitian form with matrix $I_{p}$, with $p \geq n-p$ [7, Section 7.5.3]. The number of equivalence classes of nondegenerate hermitian forms over $\mathbb{H}^{n}$ is then $\left[\frac{n}{2}\right]+1$. We fix

$$
G=\left\{M \in \mathrm{GL}(n, \mathbb{H}): \sigma(M)^{t} I_{p} M=I_{p}\right\} .
$$

The group $G$ is the group of automorphisms of the hermitian vector space $\left(\mathbb{H}^{n}, h\right)$. Hence the set of equivalence classes of nondegenerate hermitian forms over $\mathbb{H}^{n}$ is in one-to-one correspondence with the cohomology set $H^{1}(k, G(\bar{k}))$ (see [22, Chapter X, Section 2, Proposition 4] or 
[16, Section 2.6, Lemma 3]). Since the set of $K$-differential isomorphism classes of PicardVessiot extensions for $\mathcal{L}(Y)=0$ is also in one-to-one correspondence with the cohomology set $H^{1}(k, G(\bar{k}))$, we have that the number of $K$-differential isomorphism classes of Picard-Vessiot extensions for $\mathcal{L}(Y)=0$ is equal to the number of equivalence classes of nondegenerate hermitian forms over $\mathbb{H}^{n}$. Let us note that for $M, N \in M_{n}(\mathbb{H})$, we have $\sigma(M N)^{t}=\sigma(N)^{t} \sigma(M)^{t}$. We determine now the image of $G$ under the isomorphism $\mu \otimes_{k} \bar{k}: \operatorname{GL}\left(n, \mathbb{H} \otimes_{k} \bar{k}\right) \rightarrow$ GL $(2 n, \bar{k})$. For $M \in \mathrm{GL}\left(n, \mathbb{H} \otimes_{k} \bar{k}\right)$, we have $\left(\mu \otimes_{k} \bar{k}\right)\left(\sigma\left(M^{t}\right)\right)=A_{2 n}\left(\mu \otimes_{k} \bar{k}\right)(M)^{t} A_{2 n}^{-1}$, for $A_{2 n}$ the matrix $\left(a_{i j}\right)_{1 \leq i, j \leq 2 n}$ defined by

$$
a_{i j}=\left\{\begin{aligned}
1 & \text { if } i \text { is odd and } j=i+1 \\
-1 & \text { if } i \text { is even and } j=i-1 \\
0 & \text { in all other cases. }
\end{aligned}\right.
$$

Hence $\sigma(M)^{t} I_{p} M=I_{p}$ implies $\left(\mu \otimes_{k} \bar{k}\right)(M)^{t} A_{2 n}^{-1} I_{2 p}\left(\mu \otimes_{k} \bar{k}\right)(M)=A_{2 n}^{-1} I_{2 p}$. Now the group

$$
\bar{G}:=\left\{N \in \operatorname{GL}(2 n, \bar{k}): N^{t} A_{2 n}^{-1} I_{2 p} N=A_{2 n}^{-1} I_{2 p}\right\}
$$

is a conjugate form of $\operatorname{Sp}(2 n, \bar{k})$ and $\mu(G)=\left\{N \in \bar{G}: N=A_{2 n} \bar{N} A_{2 n}^{-1}\right\}$. A complete set of nonequivalent 1-cocycles of $\mathrm{Gal}(\bar{k} \mid k)$ in $\mu(G)$ is given by

$$
\begin{aligned}
x_{q}: \operatorname{Gal}(\bar{k} \mid k) & \rightarrow \mu(G), \\
c & \mapsto B_{q}:=I_{2 q} I_{2 p},
\end{aligned}
$$

with $q$ an integer, $0 \leq q \leq n, q \geq n-q$. The 1-cocycle $x_{q}$ corresponds to a Picard-Vessiot extension $L_{q}$ of $K$ for $\mathcal{L}(Y)=0$ such that there is a differential isomorphism $f_{q}$ from $L(\mathrm{i})$ to $L_{q}(\mathrm{i})$ satisfying $x_{q}=f_{q}^{-1} c\left(f_{q}\right)$. The differential isomorphism $f_{q}$ is determined by the matrix $D_{2 q}$ giving the images of a vector space of solutions. The isomorphism $f_{q}$ satisfies $x_{q}=f_{q}^{-1} c\left(f_{q}\right)$ if and only if the matrix $D_{2 q}$ satisfies $B_{q}=D_{2 q}^{-1} \overline{D_{2 q}}$. We may take $D_{2 q}:=J_{2 q} J_{2 p}$. If $N$ is a matrix belonging to $\mu(G)$, it satisfies $N^{t} A_{2 n}^{-1} I_{2 p} N=A_{2 n}^{-1} I_{2 p}$. Then the conjugate matrix $P$ of $N$ by $D_{2 q}, P:=D_{2 q} N D_{2 q}^{-1}$, satisfies $P^{t} A_{2 n}^{-1} I_{2 q} P=A_{2 n}^{-1} I_{2 q}$, hence the Picard-Vessiot extension corresponding to the 1 -cocycle $x_{q}$ has differential Galois group $\mathrm{SU}\left(n, \mathbb{H}, h_{q}\right)$, where $h_{q}$ denotes the hermitian form with index $q$.

Gathering the results in this section we may state the following theorem.

Theorem 5.1. Let $K$ be a formally real differential field with real closed field of constants $k$, $\mathcal{L}(Y)=0$ a linear differential equation defined over $K, L \mid K$ a formally real Picard-Vessiot extension for $\mathcal{L}(Y)=0$ and $G$ the differential Galois group of $L \mid K$. We assume that $G$ is a real form of $\operatorname{Sp}(2 n)$.

(1) If $G=\operatorname{Sp}(2 n, k), L \mid K$ is the unique Picard-Vessiot extension for the equation $\mathcal{L}(Y)=0$.

(2) If $G=\mathrm{SU}\left(n, \mathbb{H}, h_{p}\right)$, where $h_{p}$ is a nondegenerate hermitian form on $\mathbb{H}^{n}$, of index $p$, $0 \leq p \leq n, p \geq n-p$, there are $[n / 2]+1$ Picard-Vessiot extensions for the equation $\mathcal{L}(Y)=0$, up to $K$-differential isomorphism, and their differential Galois groups range over the whole set of groups $G=\mathrm{SU}\left(n, \mathbb{H}, h_{q}\right)$, with $h_{q}$ a nondegenerate hermitian form on $\mathbb{H}^{n}$, of index $q, 0 \leq q \leq n, q \geq n-q$.

\section{Conclusions}

In the preceding we have seen cases in which a linear differential equation $\mathcal{L}(Y)=0$ defined over a formally real differential field $K$ has Picard-Vessiot extensions which are not formally real. 
The occurrence of these extensions depends on the real form of the differential Galois group of $\mathcal{L}(Y)=0$. When the number of $K$-differential isomorphisms of Picard-Vessiot extensions of $\mathcal{L}(Y)=0$ is bigger than 1, we find several situations concerning the differential Galois group, either it is the same for all Picard-Vessiot extensions or it ranges over a subset or the whole set of real forms of the group $G$ of the formally real Picard-Vessiot extension. It would be interesting to know if, in the case when $K$ is a field of real functions, the solutions of such an equation in a non formally real differential field and the variation of the differential Galois group have some physical interpretation. Some inspiring examples in Hamiltonian mechanics are presented in [3].

\section{Acknowledgements}

Both authors acknowledge support of grant MTM2015-66716-P (MINECO/FEDER, UE). The authors thank the anonymous referees for their valuable remarks and suggestions.

\section{References}

[1] Amano K., Masuoka A., Picard-Vessiot extensions of Artinian simple module algebras, J. Algebra 285 (2005), 743-767.

[2] André Y., Différentielles non commutatives et théorie de Galois différentielle ou aux différences, Ann. Sci. École Norm. Sup. (4) 34 (2001), 685-739, arXiv:math.GM/0203274.

[3] Audin M., Exemples de hamiltoniens non intégrables en mécanique analytique réelle, Ann. Fac. Sci. Toulouse Math. (6) 12 (2003), 1-23.

[4] Bachmayr A., Harbater D., Hartmann J., Differential Galois groups over Laurent series fields, Proc. Lond. Math. Soc. 112 (2016), 455-476.

[5] Bayer-Fluckiger E., Parimala R., Classical groups and the Hasse principle, Ann. of Math. 147 (1998), 651-693, Correction, Ann. of Math. 163 (2006), 381.

[6] Bochnak J., Coste M., Roy M.-F., Real algebraic geometry, Ergebnisse der Mathematik und ihrer Grenzgebiete (3), Vol. 36, Springer-Verlag, Berlin, 1998.

[7] Borel A., Lie groups and linear algebraic groups. I. Complex and real groups, in Lie Groups and Automorphic Forms, AMS/IP Stud. Adv. Math., Vol. 37, Amer. Math. Soc., Providence, RI, 2006, 1-49.

[8] Crespo T., Hajto Z., Algebraic groups and differential Galois theory, Graduate Studies in Mathematics, Vol. 122, Amer. Math. Soc., Providence, RI, 2011.

[9] Crespo T., Hajto Z., Real Liouville extensions, Comm. Algebra 43 (2015), 2089-2093, arXiv:1206.2283.

[10] Crespo T., Hajto Z., Sowa E., Picard-Vessiot theory for real fields, Israel J. Math. 198 (2013), 75-89.

[11] Crespo T., Hajto Z., van der Put M., Real and p-adic Picard-Vessiot fields, Math. Ann. 365 (2016), 93-103, arXiv:1307.2388.

[12] Deligne P., Milne J.S., Tannakian categories, in Hodge cycles, motives, and Shimura varieties, Lecture Notes in Math., Vol. 900, Editors P. Deligne, J.S. Milne, A. Ogus, K.-Y. Shih, Springer-Verlag, Berlin - New York, 1982, 101-228.

[13] Dyckerhoff T., The inverse problem of differential Galois theory over the field $\mathbb{R}(z)$, arXiv:0802.2897.

[14] Gel'fond O.A., Hovanskii A.G., Real Liouville functions, Funct. Anal. Appl. 14 (1980), 122-123.

[15] Kamensky M., Pillay A., Interpretations and differential Galois extensions, Int. Math. Res. Not. 2016 (2016), 7390-7413.

[16] Kneser M., Lectures on Galois cohomology of classical groups, Tata Institute of Fundamental Research Lectures on Mathematics, Vol. 47, Tata Institute of Fundamental Research, Bombay, 1969.

[17] Knus M.-A., Merkurjev A., Rost M., Tignol J.-P., The book of involutions, American Mathematical Society Colloquium Publications, Vol. 44, Amer. Math. Soc., Providence, RI, 1998.

[18] Kolchin E.R., Algebraic matric groups and the Picard-Vessiot theory of homogeneous linear ordinary differential equations, Ann. of Math. 49 (1948), 1-42.

[19] León Sánchez O., Pillay A., Some definable Galois theory and examples, Bull. Symb. Log. 23 (2017), 145-159, arXiv:1511.05541. 
[20] Malle G., Testerman D., Linear algebraic groups and finite groups of Lie type, Cambridge Studies in Advanced Mathematics, Vol. 133, Cambridge University Press, Cambridge, 2011.

[21] Prestel A., Lectures on formally real fields, Lecture Notes in Math., Vol. 1093, Springer-Verlag, Berlin, 1984.

[22] Serre J.-P., Local fields, Graduate Texts in Mathematics, Vol. 67, Springer-Verlag, New York - Berlin, 1979.

[23] Serre J.-P., Cohomologie galoisienne, 5th ed., Lecture Notes in Math., Vol. 5, Springer-Verlag, Berlin, 1994.

[24] Springer T.A., Linear algebraic groups, 2nd ed., Progress in Mathematics, Vol. 9, Birkhäuser Boston, Inc., Boston, MA, 1998.

[25] Steinberg R., Lectures on Chevalley groups, Yale University, New Haven, Conn., 1968.

[26] Tits J., Classification of algebraic semisimple groups, in Algebraic Groups and Discontinuous Subgroups (Proc. Sympos. Pure Math., Boulder, Colo., 1965), Amer. Math. Soc., Providence, R.I., 1966, 33-62.

[27] van der Put M., Singer M.F., Galois theory of linear differential equations, Grundlehren der Mathematischen Wissenschaften, Vol. 328, Springer-Verlag, Berlin, 2003. 